\title{
Expression of Chemokines and Chemokine Receptors in Brain Tumor Tissue Derived Cells
}

\author{
Mahboobeh Razmkhah ${ }^{1}$, Fahimeh Arabpour ${ }^{1}$, Mousa Taghipour ${ }^{2}$, Ali \\ Mehrafshan $^{2}$, Nooshafarin Chenari ${ }^{1}$, Abbas Ghaderi ${ }^{1,3 *}$
}

\begin{abstract}
Chemokine and chemokine receptor expression by tumor cells contributes to tumor growth and angiogenesis and thus these factors may be considered as tumor markers. Here we aimed to characterize cells directly extracted from glioma, meningioma, and secondary brain tumors as well as non-tumoral cells in vitro. Cells were isolated from brain tissues using $0.2 \%$ collagenase and characterized by flow cytometry. Expression of SDF-1, CXCR4, CXCR7, RANTES, CCR5, MCP-1 and IP-10 was defined using flow cytometry and qRT-PCR methods. Brain tissue isolated cells were observed as spindle-shaped cell populations. No significant differences were observed for expression of SDF-1, CXCR4, CXCR7, RANTES, CCR5, and IP-10 transcripts. However, the expression of CXCR4 was approximately 13-fold and 110-fold higher than its counterpart, CXCR7, in meningioma and glioma cells, respectively. CXCR7 was not detectable in secondary tumors but CXCR4 was expressed. In non tumoral cells, CXCR7 had 1.3-fold higher mRNA expression than CXCR4. Flow cytometry analyses of RANTES, MCP1, IP-10, CCR5 and CXCR4 expression showed no significant difference between low and high grade gliomas. Differential expression of CXCR4 and CXCR7 in brain tumors derived cells compared to non-tumoral samples may have crucial impacts on therapeutic interventions targeting the SDF-1/CXCR4/CXCR7 axis.
\end{abstract}

Keywords: Brain tumors - chemokine - chemokine receptor - meningioma - glioma

Asian Pac J Cancer Prev, 15 (17), 7201-7205

\section{Introduction}

Brain tumor is one of the leading causes of cancer related death worldwide. Glioblastoma is ranked as the most highly aggressive brain tumor which has high expression of mediators of invasion such as angiogenic factors and chemokines (Albulescu et al., 2013).

Chemokines and chemokine receptors are constitutively expressed by microglial cells, astrocytes, and neurons and play crucial roles in central nervous system (CNS) from development to neoplastic process (Bajetto et al., 2001). Chemokines and their receptors are involved in many pathological processes including inflammation, angiogenesis, and tumor cell growth and dissemination (Bajetto et al., 2001; Bian et al., 2007). SDF-1 and its receptors, CXCR4 and CXCR7, are recognized as important prognostic factors in different types of brain tumors such as glioma and meningioma (Bian et al., 2007; Yang et al., 2007; Bajetto et al., 2007). In glioblastoma multiform, tumor grade is associated with the level of SDF-1 expression (Rempel et al., 2000). SDF-1 may act as a stimulant for proliferation and survival of glioblastoma multiform, medulablstoma cell lines and xenografted tumors (Bajetto et al., 2007).

In addition to the effects of chemokines on tumor cells, they may contribute to the recruitment of inflammatory cells to the tumor microenvironment. In this connection, SDF-1 is known as a chemoattractant for recruiting tumor associated macrophages (TAMs) (Wang et al., 2012) which are accompanied with higher grades in gliomas (Nishie et al., 1999). SDF-1 contributes to the survival of bone marrow-derived mesenchymal stem cells through PI3K/Akt and ERK1/2 signaling pathways which in turn enhance the anti-inflammatory milieu of tumor microenvironment (Zheng et al., 2007).

One of the most challenging subjects in malignant features and tumor metastases is the presence of cancer stem cells in tumor tissues. It is reported that CD133+ glioblastoma stem cells have higher expression of CXCR4 compared to CD133- cells (Liu et al., 2006).

There is data suggesting that SDF-1 can orchestrate the events of tumor microenvironment for example the signaling pathway of SDF-1/CXCR4 axis causes the upregulation of MCP-1 and IL- 8 in tumor site where they play important roles in tumor angiogenesis (Oh et al., 2001; Domanska et al., 2011). MCP-1 is reported to be upregulated in GBM and anaplastic astrocytoma and mediates the recruitment of macrophages with proangiogenic functions into tumor area in the gliomas (Leung et al., 1997; Lee et al., 2005). Dwyer et al.

${ }^{1}$ Shiraz Institute for Cancer Research, ${ }^{2}$ Department of Neurosurgery, ${ }^{3}$ Department of Immunology, School of Medicine, Shiraz University of Medical Sciences, Shiraz, Iran *For correspondence: ghaderia@sums.ac.ir 
demonstrated that IL- 8 has the ability to function as an angiogenic factor in glioblastoma (Dwyer et al., 2012). CXCR7 is reported to be upregulated in blood vessels of malignant brain tumors and mediates the recruitment of endothelial cells to the SDF-1 secreted tumor cells (Madden et al., 2004).

Here we first examined the ability of glioma and meningioma brain tumor cells to grow in culture. Then expressions of various chemokines and chemokine receptors have been further investigated in tumor cells.

\section{Materials and Methods}

\section{Subjects}

Brain tumor tissues were obtained from a total number of twenty seven Iranian patients with brain tumor, 20 cases with meningioma and 7 cases with Glioma (4 with high grade glioma and 3 with low grade glioma). The mean ages of meningioma and glioma patients were 47.5 and 38.5 years, respectively. mRNA and protein expression levels were also assessed in 4 individuals with convulsion and 2 cases with secondary brain tumor (1 with sarcoma and another with breast cancer). All patients provided their informed consent to take part in this study. Patients with chemotherapy or radiotherapy were not entered our study.

\section{Cell isolation and culture}

Separated tissues from meningioma and glioma brain tumor patients and individuals with convulsion were washed with phosphate buffered saline (PBS), minced in small pieces and digested with $0.2 \%$ collagenase type 1 (GIBCO, USA) at $37^{\circ} \mathrm{C}$ on a shaker for 30 minutes. The resulted soup was centrifuged and then the pellet including the adherent cells was resuspended in DMEM/F12 culture medium (Biosera, USA) containing 10\% fetal bovine serum (GIBCO, USA) and $1 \%$ penicillin/streptomycin (Biosera UK). Non adherent cells were discarded after 24 hrs post culture. The adherent cells were cultured by changing medium every 3 days and harvested in passage 3 after 30 days culture in order to have a homogenic population.

RNA extraction, reverse transcription and quantitative real-time PCR ( $q R T$-PCR)

Total RNA was extracted from isolated cells using TRizol (Invitrogen, Germany) and chloroform (Merck, Germany). cDNA was synthesized from the extracted RNAs using the cDNA synthesis kit based on the manufacturer's guidelines (Fermentas, Canada). The abundance of IP-10, RANTES, CCR5, SDF-1, CXCR4 and CXCR7 gene transcripts were determined using an ABI thermal cycler (ABI, USA) for qRT-PCR. Each PCR reaction was carried out in a final volume of $20 \mu \mathrm{l}$ containing $2 \mu \mathrm{l} \mathrm{cDNA}, 10 \mu \mathrm{l}$ of $2 \mathrm{X}$ SYBR Green Master Mix (Fermentas, Canada), $0.3 \mu 1$ of each 10 pmol forward and reverse primers and 7.4 41 DEPC treated water. Thermal cycling for all the genes was initiated with a denaturation step at $95^{\circ} \mathrm{C}$ for $10 \mathrm{~min}$, followed by 50 cycles with the subsequent program for each cycle: $95^{\circ} \mathrm{C}$ for $15 \mathrm{~s}, 56^{\circ} \mathrm{C}$ for $20 \mathrm{~s}$ and $60^{\circ} \mathrm{C}$ for 1 minute. All data were compared to those for beta actin housekeeping gene.
Chemokine and chemokine receptor profile by flow cytometry

Brain tumor isolated cells were harvested by treating with dissociation solution (Sigma, USA). For intracellular staining, the cells were incubated with Brefeldin (BD Biosciences, USA) for 5 hours. Afterward, cells were centrifuged and the resulted pellet was incubated with $1 \%$ cell fix and then $0.2 \%$ saponin. Next, cells were either stained with $5 \mu 1 \mathrm{PE}$-conjugated mouse anti-human RANTES, IP-10, MCP-1 or CXCR4 and FITC-conjugated mouse anti-human CCR5 antibodies (BD Biosciences, USA). After 30 minutes incubation on ice, cells were washed twice with PBS and analysis was performed on a Becton Dickinson FACS Vantage instrument and further analyzed using WinMDI 2.8 software. Isotype-matched monoclonal antibodies (BD Biosciences, USA) were used to exclude non specific staining of the cells.

\section{Statistical analysis}

The relative amounts of chemokines and chemokine receptors transcripts were determined using $2^{-\Delta \mathrm{Ct}}$ formula and were compared between different groups using nonparametric Mann-Whitney U test and kruskal-wallis $\mathrm{H}$ test by SPSS software version 15. Relative expression was plotted and evaluated by means of GraphPad Prism 5 software (Inc; San Diego CA, USA, 2003). p <0.05 was regarded as significant in all statistical analyses.

\section{Results}

\section{Phenotypic features of tumor cells}

As shown in (Figure 1), brain tumor isolated cells were appeared as plastic adherent and homogenous spindleshaped cell population with the ability to be cultured up to 11 passages.

$m R N A$ expression of chemokines and chemokine receptors by $q R T-P C R$

The mRNA expressions of SDF-1, CXCR4, CXCR7, RANTES, CCR5, and IP-10 in meningioma and glioma cells are shown in (Figure 2A and 2B). Based on the results no significant difference was observed between the expression of chemokines studied and chemokine receptors. However, SDF-1 showed the statistically significant higher expression in both meningioma and glioma cells when compared to its receptors, CXCR4 $(p<0.05)$ and CXCR7 $(p<0.0001)$. The expression of CXCR4 was approximately 13 -fold and 110 -fold higher than it's counterpart, CXCR7, in meningioma and glioma cells, respectively. CXCR7 was not detectable in secondary tumors but CXCR4 was expressed by these cells

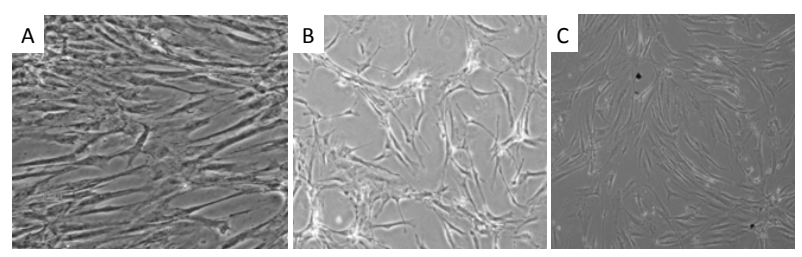

Figure 1. Spindle Shaped Cells Isolated From A. NonTumoral, B. Meningioma and C. Glioma Brain Tissues in Culture at Passage 3 
A

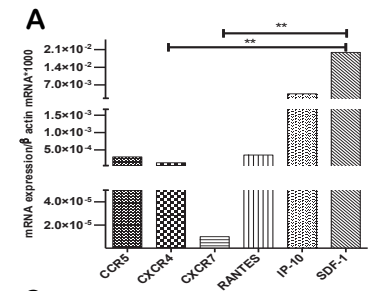

C

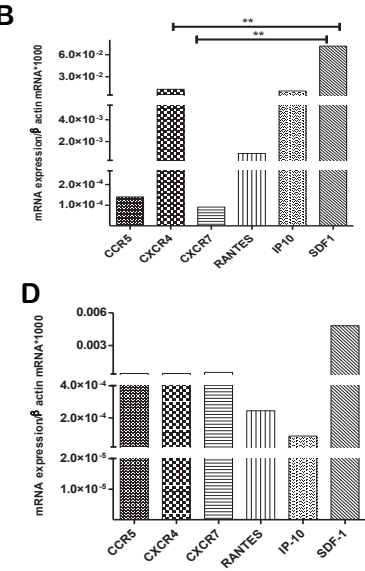

Figure 2. mRNA Expression of Chemokines and Chemokine Receptors in Cells Isolated from A) Meningioma B) Glioma C) Secondary Brain Tumors and D) Non-Tumoral Samples. Data are shown as the median of $2^{-\triangle \mathrm{CT}} \times 103$

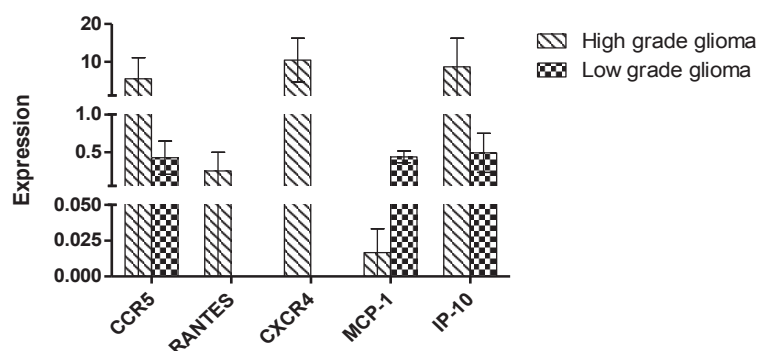

Figure 3. Expressions of RANTES, MCP-1, IP-10, CCR5 and CXCR4 in Low and High Grade Glioma Cells. Data are shown as mean \pm SEM

(Figure 2C). In non tumoral cells, CXCR7 had 1.3-fold higher mRNA expression than CXCR4 (Figure 2D).

Comparison of chemokine and chemokine receptors expression between low and high grade glioma cells

Flow cytometry analysis of RANTES, MCP-1, IP-10, CCR5 and CXCR4 expressions showed no significant difference between low and high grade gliomas. However, there were 13 -fold, and 17.7 fold higher expressions of CCR5 and IP-10 in high grade compared to low grade cells. RANTES and CXCR4 were detectable only in high grade samples. In contrast, MCP-1 showed a 24-fold higher expression in low grade compared to high grade samples (Figure 3).

\section{Discussion}

Chemokines have been known to mediate the recruitment of different types of immune cells to the tumor microenvironment. However, they have often had positive effects on tumor growth through provoking angiogenesis and metastasis. Human gliomas and astrocytomas produce several chemokines and receptors such as SDF-1, CXCR4, MCP-1, IL-8, IP-10, I-TAC and CXCL16 (Ludwig et al., 2005; Maru et al., 2008; Zhou et al., 2002). CXCR4 is expressed in areas of angiogenesis and in higher grades of gliomas which is associated with much progression of disease in the brain (Rempel et al., 2000; Stevenson et al., 2008; Komatani et al., 2009). Moreover, expression of chemokines and chemokine receptors would be of huge importance in meningiomas. Barbieri et al. have claimed that meningioma tissues express a number of receptors such as CXCR1, CXCR2, CXCR3, CXCR4, and CXCR5 among which CXCR4 had a mitogenic activity through ERK1/2 signalling pathway (Barbieri et al., 2006). CXCR7 mRNA was also detected in tumor samples from patients with neurilemmoma, pituitary adenoma, hemangioblastoma, and meningioma but the level of expression was significantly higher in neurilemmoma and meningioma compared to tissues from pituitary adenoma and hemangioblastoma (Tang et al., 2012).

Here we assessed the expressions of chemokines and chemokine receptors in glioma, meningioma, non-tumoral and brain metastasis tumor tissues. As mentioned earlier the pattern of CXCR4 and CXCR7 expressions were different between tumoral and non-tumoral samples because in tumoral samples CXCR4 had much more mRNA expression compared to CXCR7 while in nontumoral samples CXCR7 had higher expression. Thus, higher expression of CXCR4 may cause the aggressive behaviour of brain tumor cells compared to non-tumoral cells. These data showed why increasing attention has recently been directed to address new therapeutic approaches using SDF-1/CXCR4 axis in different types of tumors (Teicher and Fricker, 2010).

Consistently it has been reported that CXCR4 and CXCR7 receptors are different in both expression patterns and functional roles in Neuroblastoma (Liberman et al., 2012). These variations were also addressed in meningiomas. CXCR 4 has been reported to be expressed inside the tumor cells while CXCR7 was expressed in tumor endothelial cells; thereby CXCR7 is thought to be involved in vascularization of meningioma (Würth et al., 2011). It has previously been reported by Hattermann et al. that both CXCR4 and CXCR7 are expressed in astrocytomas/glioblastomas but CXCR4 is found on stemlike cells whereas CXCR7 detected on tumor-infiltrating microglial cells. They concluded that CXCR4 is likely to contribute to the recruitment of tumor stem like cells while CXCR7 seems to inhibit the apoptosis of glioma cells (Hattermann et al., 2010).

Comparison of chemokine and chemokine receptors expression between low and high grade glioma cells showed higher expressions of RANTES, IP-10, CXCR4 and CCR5, but not MCP-1, in high grade compared to low grade samples. These results predict that for disease progression in glioma, RANTES/CCR5, SDF-1/CXCR4 and IP-10 may play more crucial roles compared to MCP1. This finding about CXCR4 is further supported by a recent study showing that CXCR4 is overexpressed by invasive compared to non-invasive human glioma cells (Ehtesham et al., 2006 ; Sciumè et al., 2010).

Studies on brain tissues have generated considerable interest because a number of researchers showed that brain derived cells may have mesenchymal stem cell (MSC) properties. U87MG cell line as a human GBM cell presents MSC properties both phenotypicaly and functionally. These cells express distinctive MSC markers and have the ability to be differentiated to divergent cell lineages. It is predicted that these MSC like cells have 
high resemblance to MSCs which are derived from other sources such as adipose or bone marrow since both express low levels of HLA-DR. Therefore they expected to have immunomodulatory characteristics which may enhance their tumorigenicity in vivo. Consistently, general features of glioblastoma cells with immune suppression were observed after using U87MG cell line in immunocompetent rats (Nakahata et al., 2010). In the present study which the cells were directly isolated from the brain tissues, we also found both brain tumor cells and non-tumoral cells as mesenchymal stem cell like cells with spindle shape in culture. Interestingly, these cells have the ability to grow in high passages. Similarly, in a study by Brewer et al. cells from meningioma, glioblastoma and secondary tumors (metastasis) were cultured in defined mediums and appeared with mesenchymal morphology (Brewer and LeRoux, 2007).

To sum up, CXCR4 might have much more important functional roles in brain tumor growth compared to CXCR7, considering the higher expression of this receptor in glioma, meningioma and metastatic tumor cells than non-tumoral samples. Such conception may guide the improvement of the clinical approaches in different types of brain tumors but further studies with a larger sample size are undoubtedly needed. Additionally, assessment the immunomodulatory characteristics of brain derived cells will be helpful for elucidating the actual role of these cells.

\section{Acknowledgements}

The authors thank patients for their kind contribution in this project. This work was supported by grants from Shiraz University of Medical Sciences (Grant No. 895290) and Shiraz Institute for Cancer Research (ICR-100504). There is no conflict of interest stated.

\section{References}

Albulescu R, Codrici E, Popescu ID, et al (2013). Cytokine patterns in brain tumour progression. Mediators Inflamm, 2013, 979748.

Bajetto A, Barbieri F, Pattarozzi A, et al (2007). CXCR4 and SDF1 expression in human meningiomas: a proliferative role in tumoral meningothelial cells in vitro. Neuro Oncol, 9,3-11.

Bajetto A, Bonavia R, Barbero S, Florio T, Schettini G (2001). Chemokines and their receptors in the central nervous system. Front Neuroendocrinol, 22, 147-84.

Barbieri F, Bajetto A, Porcile C, et al (2006). CXC receptor and chemokine expression in human meningioma: SDF1/CXCR4 signaling activates ERK $1 / 2$ and stimulates meningioma cell proliferation. Ann N Y Acad Sci, 1090, 332-43.

Bian XW, Yang SX, Chen JH, et al (2007). Preferential expression of chemokine receptor CXCR4 by highly malignant human gliomas and its association with poor patient survival. Neurosurgery, 61, 570-8.

Brewer GJ and LeRoux PD (2007). Human primary brain tumor cell growth inhibition in serum-free medium optimized for neuron survival. Brain Res, 1157, 156-66.

Domanska UM, Kruizinga RC, den Dunnen WF, et al (2011). The chemokine network, a newly discovered target in high grade gliomas. Crit Rev Oncol Hematol, 79, 154-63.

Dwyer J, Hebda JK, Le Guelte A, et al (2012). Glioblastoma cell-secreted interleukin-8 induces brain endothelial cell permeability via CXCR2. PLoS One, 7, 45562.

Ehtesham M, Winston JA, Kabos P, Thompson RC (2006). CXCR4 expression mediates glioma cell invasiveness. Oncogene, 25, 2801-6.

Hattermann K, Held-Feindt J, Lucius R, et al (2010). The chemokine receptor CXCR7 is highly expressed in human glioma cells and mediates antiapoptotic effects. Cancer Res, 70, 3299-308.

Komatani H, Sugita Y, Arakawa F, Ohshima K, Shigemori M (2009). Expression of CXCL12 on pseudopalisading cells and proliferating microvessels in glioblastomas: an accelerated growth factor in glioblastomas. Int $\mathrm{J} \mathrm{Oncol,}$ 34, 665-72.

Lee S, Jilani S, Nikolova GV, Carpizo D, Iruela-Arispe ML (2005). Processing of VEGF-A by matrix metalloproteinases regulates bioavailability and vascular patterning in tumors. J Cell Biol, 169, 681-91.

Leung SY, Wong MP, Chung LP, Chan AS, Yuen ST (1997). Monocyte chemoattractant protein-1 expression and macrophage infiltration in gliomas. Acta Neuropathol, 93, 518-27.

Liberman J, Sartelet H, Flahaut M, et al (2012). Involvement of the CXCR7/CXCR4/CXCL12 axis in the malignant progression of human neuroblastoma. PLoS One, 7, 43665.

Liu G, Yuan X, Zeng Z, et al (2006). Analysis of gene expression and chemoresistance of CD133+ cancer stem cells in glioblastoma. Mol Cancer, 5, 61 .

Ludwig A, Schulte A, Schnack C, et al (2005). Enhanced expression and shedding of the transmembrane chemokine CXCL16 by reactive astrocytes and glioma cells. $J$ Neurochem, 93, 1293-303.

Madden SL, Cook BP, Nacht M, et al (2004). Vascular gene expression in nonneoplastic and malignant brain. Am J Pathol, 165, 601-8.

Maru SV, Holloway KA, Flynn G, et al (2008). Chemokine production and chemokine receptor expression by human glioma cells: role of CXCL10 in tumour cell proliferation. J Neuroimmunol, 199, 35-45.

Nakahata AM, Suzuki DE, Rodini CO, et al (2010). Human glioblastoma cells display mesenchymal stem cell features and form intracranial tumors in immunocompetent rats. $J$ Stem Cells, 5, 103-11.

Nishie A, Ono M, Shono T, et al (1999). Macrophage infiltration and hemeoxygenase- 1 expression correlate with angiogenesis in human gliomas. Clin Cancer Res, 5, 1107-13.

Oh JW, Drabik K, Kutsch O, et al (2001). CXC chemokine receptor 4 expression and function in human astroglioma cells. J Immunol, 166, 2695-704.

Rempel SA, Dudas S, Ge S, Gutiérrez JA (2000). Identification and localization of the cytokine SDF1 and its receptor, CXC chemokine receptor 4 , to regions of necrosis and angiogenesis in human glioblastoma. Clin Cancer Res, 6 , 102-11.

Sciumè G, Santoni A, Bernardini G (2010). Chemokines and glioma: invasion and more. J Neuroimmunol, 224, 8-12.

Stevenson CB, Ehtesham M, McMillan KM, et al (2008). CXCR4 expression is elevated in glioblastoma multiforme and correlates with an increase in intensity and extent of peritumoral T2-weighted magnetic resonance imaging signal abnormalities. Neurosurgery, 63, 560-9.

Tang T, Xia QJ, Chen JB, Xi MR, Lei D (2012). Expression of the CXCL12/SDF-1 chemokine receptor CXCR7 in human brain tumours. Asian Pac J Cancer Prev, 13, 5281-6.

Teicher BA and Fricker SP (2010). CXCL12 (SDF-1)/CXCR4 pathway in cancer. Clin Cancer Res, 16, 2927-31.

Wang SC, Hong JH, Hsueh C, Chiang CS (2012). Tumor- 
secreted SDF-1 promotes glioma invasiveness and TAM tropism toward hypoxia in a murine astrocytoma model. Lab Invest, 92, 151-62.

Würth R, Barbieri F, Bajetto A, et al (2011). Expression of CXCR7 chemokine receptor in human meningioma cells and in intratumoral microvasculature. J Neuroimmunol, 234, 115-23.

Yang YC, Lee ZY, Wu CC, et al (2007). CXCR4 expression is associated with pelvic lymph node metastasis in cervical adenocarcinoma. Int J Gynecol Cancer, 17, 676-86.

Zheng H, Fu G, Dai T, Huang H (2007). Migration of endothelial progenitor cells mediated by stromal cell-derived factor1alpha/CXCR4 via PI3K/Akt/eNOS signal transduction pathway. J Cardiovasc Pharmacol, 50, 274-280.

Zhou Y, Larsen PH, Hao C, Yong VW (2002). CXCR4 is a mayor chemokine receptor on glioma cells and mediates their survival. J Biol Chem, 277, 49481-7. 